\title{
Characterizing Norovirus Transmission from Outbreak Data, United States
}

\author{
Molly K. Steele, Mary E. Wikswo, Aron J. Hall, Katia Koelle, \\ Andreas Handel, Karen Levy, Lance A. Waller, Ben A. Lopman
}

Norovirus is the leading cause of acute gastroenteritis outbreaks in the United States. We estimated the basic $\left(R_{0}\right)$ and effective $\left(R_{e}\right)$ reproduction numbers for 7,094 norovirus outbreaks reported to the National Outbreak Reporting System (NORS) during 2009-2017 and used regression models to assess whether transmission varied by outbreak setting. The median $R_{0}$ was 2.75 (interquartile range [IQR] 2.38-3.65), and median $R_{e}$ was 1.29 (IQR 1.12-1.74). Long-term care and assisted living facilities had an $\mathrm{R}_{0}$ of 3.35 (95\% Cl 3.26-3.45), but $\mathrm{R}_{0}$ did not differ substantially for outbreaks in other settings, except for outbreaks in schools, colleges, and universities, which had an $\mathrm{R}_{0}$ of 2.92 (95\% Cl 2.82-3.03). Seasonally, $\mathrm{R}_{0}$ was lowest (3.11 [95\% Cl 2.97-3.25]) in summer and peaked in fall and winter. Overall, we saw little variability in transmission across different outbreaks settings in the United States.

$\mathrm{N}$ orovirus is the most common cause of outbreaks of acute gastroenteritis (AGE) in the United States $(1,2)$. The Centers for Disease Control and Prevention (CDC) collects data on AGE outbreaks through the National Outbreak Reporting System (NORS). During 2009-2017, norovirus was the suspected or confirmed etiology of $47 \%$ of AGE outbreaks reported to NORS (3). The size and severity of outbreaks varies across different settings, times of year, and genotypes, suggesting norovirus transmissibility is variable across different outbreak settings and contexts (4). Generally, the transmission potential of infectious diseases is influenced by the infectiousness of the pathogen, the duration of infectiousness, and the number of susceptible contacts exposed during the infectious period (5).

Author affiliations: Emory University, Atlanta, Georgia, USA

(M.K. Steele, A.J. Hall, K. Koelle, K. Levy, L.A. Waller,

B.A. Lopman); Centers for Disease Control and Prevention,

Atlanta (M.E. Wikswo, A.J. Hall); University of Georgia, Athens,

Georgia, USA (A. Handel)

DOI: https://doi.org/10.3201/eid2608.191537
The reproduction number is a metric for quantifying transmissibility of a pathogen. The basic reproduction number $\left(R_{0}\right)$ is the average number of secondary cases that arise from a primary case in a completely susceptible population. The effective reproduction number $\left(R_{e}\right)$ quantifies the average number of secondary cases that arise from a primary case in a population that is not completely susceptible. $R_{e}$ varies over the course of an outbreak as the proportion of the susceptible population changes $(6,7) \cdot R_{0}$ and $R_{e}$ are not just metrics of the biologic properties of pathogens but also measures of the transmissibility of a pathogen within a specific population or setting $(8,9)$.

Several transmission modeling studies in different settings have estimated $R_{0}$ and $R_{e}$ of norovirus, but a large variation in these estimates occurs and $R_{0}$ ranges from 1.1-7.2 (10). Much of the $\mathrm{R}_{0}$ variation likely is due to differences in the structures, population mixing assumptions, and data between transmission models in different settings (10). Generally, model estimates from community surveillance data result in an $\mathrm{R}_{0}$ of $\approx 2$, but estimates from outbreak data tend to be higher and more variable. The variability of estimates from models that use outbreak data likely are driven by context; outbreaks might occur in populations that are not representative of the population as a whole and transmission likely is higher in these settings than in the community (4).

We estimated $R_{0}$ and $R_{e}$ for thousands of norovirus outbreaks in the United States. We evaluated whether $R_{0}$ was associated with setting, season, year, or geographic region. In addition, we assessed whether norovirus was suspected or confirmed as the cause of the outbreak.

\section{Methods}

\section{Data}

We obtained data on all norovirus outbreaks during 2009-2017 from NORS and CaliciNet (11). We defined 
an outbreak as $\geq 2$ epidemiologically linked cases of suspected or laboratory-confirmed norovirus. NORS data consist of web-based reports of all foodborne, waterborne, and enteric disease outbreaks transmitted by contact with environmental sources, infected persons or animals, or unknown modes of transmission reported by state, local, and territorial public health agencies. This web-based reporting system collects epidemiologic information, including the dates; settings, such as long-term care facilities, child daycare facilities, hospitals, and schools; geographic location of the outbreak; the estimated total number of cases; and exposed population (2). For settings that report staff and guest case numbers, we included these data in the estimated total number of cases and exposed population. CaliciNet data consists of sequence-derived genotypes and epidemiologic data from norovirus outbreaks submitted from local, state, and federal public health laboratories. We obtained CaliciNet genotypes that were linked to outbreak data we acquired from NORS.

For all outbreaks reported to NORS, data are collected on the total estimated primary cases, including all laboratory-confirmed and suspected primary cases. These data exclude cases associated with secondary illnesses, such as person-to-person norovirus transmission in households after a restaurant-based outbreak. However, data for calculating attack rates, specifically the number of exposed persons and the subset of the exposed persons who became ill, are only collected for outbreaks with person-to-person, environmental, or unknown transmission modes. In addition, data collected from outbreaks might not be documented consistently across a report. For example, outbreaks for which setting-specific information on the total number of guests and staff that are reported to be ill, referred to as total ill, might not match the reported total estimated primary cases. During 20092017 , a total of 17,822 suspected and confirmed norovirus outbreaks were reported to NORS. We excluded 10,728 outbreaks based on the following criteria, which we imposed hierarchically: transmission was not person-to-person $(n=3,866)$; the outbreak exposure occurred in multiple states $(n=8)$; the outbreak occurred in Puerto Rico ( $n=3)$, which we excluded because of small sample size; the size of total exposed population or major setting were not reported $(\mathrm{n}=$ $5,573)$; the total estimated primary cases and the total ill among the exposed population were not equal ( $\mathrm{n}=$ 1,231); or the total estimated primary cases or the total ill among the exposed population were reported to be greater than the total exposed population size $(n=47)$ (Appendix Figure 1, https://wwwnc.cdc.gov/EID/
article/26/8/19-1537-App1.pdf). In all, 7,094 norovirus outbreaks met our inclusion criteria in subsequent analyses (Appendix Table 1). We did not use imputation techniques to infer values for missing data because no good proxy variables inferred missing data for major settings and exposed population size.

\section{Estimating $\mathbf{R}_{\mathbf{0}}$ and $\mathbf{R}_{\mathrm{e}}$}

We used the final size method to calculate $R_{0^{\prime}}, R_{\mathrm{e}^{\prime}}$ and associated SEs (12; Appendix). The final size method calculates $R_{0}$ and $R_{e}$ based on 3 variables: the total population size of the outbreak $(N)$, the total number of cases in the outbreak (C), and the number of susceptible persons at the start of the outbreak (S). In our calculations, $C$ was informed by NORS outbreak data for the estimated total number ill and $N$ by the exposed population. NORS data does not include nor can it inform the number of susceptible persons at the start of an outbreak. Therefore, to estimate $S$, we used norovirus challenge study data on the percent of persons that become infected and develop AGE after challenge with virus. Across all published studies, the weighted average of participants in whom gastroenteritis developed after challenge is $47 \%$ (range 27\%-80\%; Appendix Table 1) (13-19). We assumed $S$ is the number of persons susceptible to disease, as opposed to infection. To calculate $S$, we multiplied $47 \%$ by $N$ and rounded to the nearest integer. For 890 outbreaks, the total number of cases, $C$, was greater than our estimated $S$; for these outbreaks we set $S$ equal to $C$, corresponding to a $100 \%$ attack rate. We also calculated $S$ assuming $27 \%$ and $80 \%$ of $N$ were susceptible to assess the sensitivity of our model results to this parameter.

\section{Regression Analysis}

After estimating $R_{0^{\prime}}, R_{e^{\prime}}$ and associated SEs for each norovirus outbreak, we fit a linear regression model to the log-transformed estimated reproduction numbers to assess whether outbreak setting, census region, season, year, suspected or confirmed norovirus, or genotype were associated with transmissibility. All variables were categorical, where the reference was assigned as the group with the most outbreaks reported, except for the suspected or confirmed variable, for which we set the referent to outbreaks with confirmed norovirus etiology. We used weighted least squares combined with estimated standard errors to produce robust estimates accounting for heteroscedasticity and non-normally distributed model residuals by using the estimatr package in $R$ version 3.4.2 $(20,21)$. We included the following variables in our models: outbreak setting; census region; 
meteorological season, defined as spring (March 1May 31), summer (June 1-August 31), fall (September 1-November 30), or winter (December 1-February 28); year, defined as July-June; whether norovirus was suspected or confirmed; and norovirus genotype, categorized as GI, GII.4, or GII.non4.

For outbreaks for which we calculated $R_{0}$ and $R_{e^{\prime}}$ we had norovirus genotype data for only $22 \%(1,571)$. In a preliminary analysis, we fit a univariate linear regression model to estimate $R_{0}$ by norovirus genotype alone and by norovirus genotype and year and found no evidence for variation (Appendix). Given these results and the small sample size, we did not include norovirus genotype in our models and performed model selection on the remaining variables. To determine which variables to include, we used a forward selection process and selected the model with the lowest Akaike information criterion and Bayes information criterion values.

\section{Sensitivity Analysis}

We tested the sensitivity of our regression model results to different modeling approaches and different assumptions of the percent susceptible at the start of an outbreak. We also fit a logistic regression model of binary transmission and a negative binomial regression of the final outbreak size by using the logtransformed exposed population size as a measure of the attack rate of an outbreak. Thus, we could make comparisons between the models to see if the results from modeling continuous transmission were consistent with the results of modeling binary transmission and attack rates. In addition, we ran all the regression models again using the assumption that $27 \%$ and $80 \%$ susceptible at the start of an outbreak, which corresponds to the minimum and maximum percent susceptible to AGE from published challenge studies (Appendix Table 2).

\section{Results}

Of the 7,094 norovirus outbreaks included in our final dataset, $75 \%(5,335)$ occurred in long-term care and assisted living facilities and 57\% $(4,016)$ occurred in winter. The median outbreak size was 28 cases (interquartile range [IQR] 16-47) and the median attack rate was $22 \%$ (IQR $11 \%-36 \%$ ) (Table 1). The median $\mathrm{R}_{0}$ was 2.75 (IQR 2.38-3.65) and the median $\mathrm{R}_{\mathrm{e}}$ was 1.29 (IQR 1.12-1.74).

\section{Model Selection and Regression Analysis}

The final selected model included the following variables: major setting, census region, season, year, and whether norovirus was suspected or confirmed
(Akaike information criterion $=5,803$; Bayes information criterion $=5,968)$ (Appendix Table 3). For long-term care and assisted living facilities, $R_{0}$ was 3.35 (95\% CI 3.26-3.45). $R_{0}$ for outbreaks in all other settings did not differ substantially, except for outbreaks in schools, colleges, and universities, in which $\mathrm{R}_{0}$ was slightly reduced, 2.92 (95\% CI 2.82-3.03) (Table 2; Appendix Figure 2). We found that $\mathrm{R}_{0}$ differed substantially by outbreak status; suspected norovirus outbreaks had a lower $\mathrm{R}_{0}, 3.02$ (95\% CI 2.94-3.10), than that for confirmed outbreaks $\left(\mathrm{R}_{0}=3.35[95 \% \mathrm{CI}\right.$ 3.26-3.45]).

Estimated $\mathrm{R}_{0}$ varied only slightly by census region and was lowest in the northeast $\left(R_{0}=3.00[95 \%\right.$ CI 2.92-3.08]). Season and year also contributed to changes in the $R_{0}$. Estimated $R_{0}$ was highest in winter (3.35 [95\% CI 3.26-3.45]) and fall (3.37 [95\% CI 3.24-3.50]) and lowest during the summer months (3.11 [95\% CI 2.97-3.25]). Outbreaks reported during January 2009-June 2012 all had higher estimated $R_{0}$ (range for individual seasonal years 3.77-3.93) than the reference period, July 2016-June 2017 (Table 2; Appendix Figure 2). Our findings were generally robust to assumptions about the proportion susceptible at the start of the outbreak and whether we modeled the outcome of $R_{0^{\prime}} R_{e^{\prime}}$ or final outbreak size (Appendix Tables 4-6, Figure 3).

\section{Discussion}

By using a large national outbreak dataset, we investigated transmission patterns of norovirus outbreaks. Our analysis led to several key findings. First, reported norovirus outbreaks in the United States have modest $\mathrm{R}_{0}\left(2.75\right.$ [IQR 2.38-3.65]) and $\mathrm{R}_{\mathrm{e}}$ (1.29 [IQR 1.12-1.74]) values. Second, we found that $R_{0}$ and $R_{e}$ did not vary across most settings, except for outbreaks in schools, colleges, and universities, which had lower estimated transmission values. Third, we found higher transmission in laboratory-confirmed outbreaks relative to suspected outbreaks and higher transmission for outbreaks occurring in the winter months relative to summer months.

Our finding that norovirus outbreaks in the United States have modest transmission values is somewhat surprising. In a recent review of norovirus modeling studies, Gaythorpe et al. (10) found $\mathrm{R}_{0}$ estimates for norovirus were 1.1-7.2. Of note, $R_{0}$ and $\mathrm{R}_{\mathrm{e}}$ estimates from transmission modeling studies that analyzed data from norovirus outbreaks were high, but variability between studies was high; $\mathrm{R}_{\mathrm{e}}$ estimates were $\approx 1-14$ (22-24). Our estimates are within the reproduction numbers estimated by using transmission models of norovirus based on outbreak data $(22,25)$. 
Table 1. Norovirus outbreaks with exposed population size reported to the National Outbreak Reporting System, United States, 2009$2017^{*}$

\begin{tabular}{|c|c|c|c|c|}
\hline Characteristics & No. $(\%)$ & $\begin{array}{c}\text { Median attack rate, } \\
\%(I Q R)\end{array}$ & $\begin{array}{c}\text { Median final size, } \\
\%(I Q R)\end{array}$ & Median $\mathrm{R}_{0}(\mathrm{IQR})$ \\
\hline All outbreaks & $7,094(100)$ & $22(11-36)$ & $28(16-47)$ & $2.75(2.38-3.65)$ \\
\hline \multicolumn{5}{|l|}{ Major setting } \\
\hline Child day care & $272(4)$ & $21(13-36)$ & $18(11-29)$ & $2.67(2.39-3.60)$ \\
\hline Hospital or healthcare facility & $271(4)$ & $22(11-38)$ & $19(11-34)$ & $2.70(2.33-3.59)$ \\
\hline Long-term care or assisted living facility & $5,335(75)$ & $23(13-36)$ & $30(17-47)$ & $2.81(2.42-3.76)$ \\
\hline Other & $350(5)$ & $20(10-36)$ & $24(15-40)$ & $2.66(2.35-3.60)$ \\
\hline Private home or residence & $42(1)$ & $66(50-91)$ & $9(6-16)$ & $3.80(2.26-4.92)$ \\
\hline Restaurant & $77(1)$ & $50(27-64)$ & $10(6-16)$ & $3.12(2.53-4.31)$ \\
\hline School, college, or university & $747(11)$ & $12(6-24)$ & $42(19-80)$ & $2.41(2.24-2.92)$ \\
\hline \multicolumn{5}{|l|}{ Season } \\
\hline Winter & $4,016(57)$ & $22(12-36)$ & $30(17-51)$ & $2.80(2.40-3.77)$ \\
\hline Fall & $808(11)$ & $21(11-37)$ & $26(15-47)$ & $2.72(2.36-3.63)$ \\
\hline Spring & $1,964(28)$ & $20(11-35)$ & $27(15-44)$ & $2.69(2.37-3.57)$ \\
\hline Summer & $306(4)$ & $17(9-33)$ & $19(11-32)$ & $2.57(2.29-3.33)$ \\
\hline \multicolumn{5}{|l|}{ Outbreak status } \\
\hline Confirmed & $3,114(44)$ & $26(15-40)$ & $35(20-55)$ & $2.99(2.51-4.22)$ \\
\hline Suspected & $3,980(56)$ & $18(9-31)$ & $24(14-40)$ & $2.59(2.32-3.27)$ \\
\hline \multicolumn{5}{|l|}{ Census region } \\
\hline Northeast & 1,898 (27) & $17(9-29)$ & $31(17-53)$ & $2.58(2.30-3.23)$ \\
\hline Midwest & $2,205(31)$ & $25(13-39)$ & $26(15-44)$ & $2.87(2.44-3.98)$ \\
\hline South & $2,224(31)$ & $23(12-38)$ & $29(17-47)$ & $2.81(2.39-3.93)$ \\
\hline West & $767(11)$ & $21(13-34)$ & $28(16-44)$ & $2.75(2.42-3.57)$ \\
\hline \multicolumn{5}{|l|}{ Year } \\
\hline 2009 Jan-Junt & $243(3)$ & $28(15-42)$ & $35(20-55)$ & $3.09(2.50-4.56)$ \\
\hline 2009 Jul-2010 Jun & $275(4)$ & $29(15-45)$ & $35(19-57)$ & $3.17(2.51-4.77)$ \\
\hline 2010 Jul-2011 Jun & $592(8)$ & $29(16-44)$ & $32(19-54)$ & $3.12(2.54-4.58)$ \\
\hline 2011 Jul-2012 Jun & 679 (10) & $27(15-40)$ & $35(19-59)$ & $3.01(2.52-4.29)$ \\
\hline 2012 Jul-2013 Jun & 967 (14) & $21(12-36)$ & $28(16-46)$ & $2.73(2.38-3.61)$ \\
\hline 2013 Jul-2014 Jun & $913(13)$ & $20(11-33)$ & $29(18-51)$ & $2.68(2.38-3.45)$ \\
\hline 2014 Jul-2015 Jun & $941(13)$ & $21(11-35)$ & $28(16-46)$ & $2.74(2.3-3.61)$ \\
\hline 2015 Jul-2016 Jun & $1,007(14)$ & $17(9-32)$ & $25(14-42)$ & $2.57(2.31-3.29)$ \\
\hline 2016 Jul-2017 Jun & $1,070(15)$ & $19(10-31)$ & $26(14-42)$ & $2.63(2.33-3.24)$ \\
\hline 2017 Jul-Dec† & $407(6)$ & $20(10-34)$ & $22(14-38)$ & $2.66(2.33-3.55)$ \\
\hline
\end{tabular}

${ }^{*} \mathrm{QQR}$, interquartile range.

†Partial norovirus years included in this analysis. The National Outbreak Reporting System was established in January 2009, and the first year of this analysis is 2009 January-June. At the time of analysis, we received data through December 2017.

However, our estimates are higher than those from several studies that estimated reproduction numbers by using population-level transmission models $(26-$ $29)$, suggesting that transmission of norovirus in outbreak settings is higher than sporadic transmission in the community.

From our main analysis, we found that outbreaks in schools, colleges, and universities had lower estimated transmission, but transmission varied little across all other settings. Relative to outbreaks in longterm care and assisted living facilities, outbreaks that occurred in private homes or residences and restaurants had higher final sizes, and schools, colleges, and universities had lower estimated attack rates. Our finding that outbreaks in the winter had higher estimated transmissibility than outbreaks that occurred in summer is likely a factor of the strong wintertime seasonality of noroviruses in the United States $(30,31)$. Consistent with this finding are the observations that norovirus case and outbreak reports are inversely correlated with temperature $(30,31)$ and that survival of norovirus surrogate viruses, such as murine norovirus and feline calicivirus, declines with increasing temperatures $(32,33)$.

Several differences we found might be driven by surveillance biases rather than differences in norovirus transmission. Suspected norovirus outbreaks without a laboratory-confirmed outbreak etiology had lower transmission than laboratory-confirmed norovirus outbreaks, perhaps because suspected outbreaks are not investigated as well as confirmed outbreaks and have lower rates of case ascertainment. Outbreaks reported in the south had higher estimated $R_{0}$ and $R_{e}$ relative to outbreaks in the northeast, which might be related to differences in the quality of reporting between these regions. For example, if surveillance in certain regions only captured larger, more easily detectable outbreaks with higher attack rates, this could bias our estimates of transmissibility upwards. Tremendous variability exists in outbreak reporting between states, $\approx 100$-fold difference between the highest and lowest reporting states, which 
likely affects the observed outbreak characteristics we included (34). Similarly, NORS has been collecting outbreak reports since January 2009, but in August 2012 CDC began a concerted effort to improve norovirus outbreak reporting to NORS and CaliciNet with the introduction of NoroSTAT $(35,36)$. Thus, our finding that norovirus outbreaks reported before August 2012 were larger and had higher estimated $R_{0}$ and $R_{e}$ values might be related to CDC's efforts to capture outbreaks that previously would not have been reported, such as smaller outbreaks. Further, because the transmission mode can be difficult to identify for norovirus outbreaks, our analysis might have included outbreaks for which the mode of transmission was misclassified as person-to-person. Larger outbreaks with higher transmission are more likely to be reported, and our results might not reflect transmission in smaller outbreaks. In addition, the exposed population size is difficult to quantify and is not consistently reported to NORS. Thus, the differences we found in estimated attack rates across different settings could

Table 2. Estimated log-linear change in $R_{0}$ from the intercept for linear regression of log transformed $R_{0}$ for norovirus outbreaks reported to the National Outbreak Reporting System, United States, 2009-2017

\begin{tabular}{|c|c|}
\hline Category & $\begin{array}{l}\text { Estimated log-linear } \\
\text { change in } \mathrm{R}_{0}(95 \% \mathrm{Cl})\end{array}$ \\
\hline Intercept & $3.35(3.26-3.45)$ \\
\hline \multicolumn{2}{|l|}{ Major setting } \\
\hline $\begin{array}{l}\text { Long-term care or assisted living } \\
\text { facility }\end{array}$ & Referent \\
\hline Child day care & $0.99(0.95-1.03)$ \\
\hline Hospital or healthcare facility & $0.93(0.90-0.97)$ \\
\hline Other & $0.97(0.93-1.01)$ \\
\hline Private home or residence & $0.99(0.82-1.19)$ \\
\hline Restaurant & $1.01(0.91-1.11)$ \\
\hline School, college, or university & $0.87(0.85-0.89)$ \\
\hline \multicolumn{2}{|l|}{ Season } \\
\hline Winter & Referent \\
\hline Fall & $1.00(0.98-1.03)$ \\
\hline Spring & $0.98(0.96-1.00)$ \\
\hline Summer & $0.93(0.89-0.96)$ \\
\hline \multicolumn{2}{|l|}{ Outbreak status } \\
\hline Confirmed & Referent \\
\hline Suspected & $0.90(0.88-0.92)$ \\
\hline \multicolumn{2}{|l|}{ Census region } \\
\hline South & Referent \\
\hline Northeast & $0.89(0.87-0.91)$ \\
\hline Midwest & $1.00(0.97-1.02)$ \\
\hline West & $0.98(0.95-1.01)$ \\
\hline \multicolumn{2}{|l|}{ Year } \\
\hline 2009 Jan-Jun & $1.16(1.10-1.23)$ \\
\hline 2009 Jul-2010 Jun & $1.17(1.11-1.23)$ \\
\hline 2010 Jul-2011 Jun & $1.16(1.12-1.21)$ \\
\hline 2011 Jul-2012 Jun & $1.12(1.08-1.16)$ \\
\hline 2012 Jul-2013 Jun & $1.04(1.01-1.07)$ \\
\hline 2013 Jul-2014 Jun & $1.02(0.99-1.06)$ \\
\hline 2014 Jul-2015 Jun & $1.05(1.02-1.08)$ \\
\hline 2015 Jul-2016 Jun & 1.02 (0.99-1.05) \\
\hline 2016 Jul-2017 Jun & Referent \\
\hline 2017 Jul-Dec & $1.04(1.00-1.09)$ \\
\hline
\end{tabular}

be due to true variability in the exposed population size across settings or variability in the reliable reporting of the exposed population size. However, our analysis restricted to outbreaks in long-term care and assisted living facilities found the same trends among the variables for outbreak status, census region, season, and year as our analysis of all outbreaks, which suggests the results are robust.

Our study has several additional limitations. First, our process of data selection might have introduced bias into our analyses. We excluded outbreaks that occurred in multiple states, which are likely to have higher transmissibility given the larger geographic range involved; however, only 8 multistate outbreaks occurred during the study period, thus the bias is likely negligible. A substantial proportion of the dataset, 5,573 (31\%) outbreaks, had to be excluded because the exposed population size was not reported. Excluding these outbreaks could introduce bias if the exposed population size is more likely to be reported for outbreaks with smaller, or larger, exposed population sizes. We only included outbreaks with person-to-person transmission; thus, our estimates of transmissibility are not generalizable to norovirus outbreaks where transmission occurs via other modes, such as foodborne, waterborne, or environmental transmission.

A second set of limitations relates to the final size method. This method assumes a susceptibleinfected-recovered type infection in a homogenously mixing population (12), but this simplification likely does not reflect true mixing patterns. In addition, we might observe different mixing patterns in each of the different outbreak settings, such as older persons in long-term care facilities versus young children in childcare. The final size method also underestimates reproduction numbers for outbreaks with high attack rates. For example, in private homes, attack rates are high, but exposed population sizes are small. If everyone in the household is infected, then no additional infections can occur in the home. Thus, the final size method cannot capture any additional transmission that could have happened if the exposed population size had been larger, such as a higher number of persons in the household. Becker termed this limitation the "wasted infection potential" (37). Further, the final size method does not account for the effect of control measures. For some of the outbreaks represented in our dataset, control measures were most likely implemented, such as isolating ill persons and cleaning contamination. Such interventions likely would reduce the number ill, and the estimated $R_{0}$ would be lower than the $R_{0}$ in the absence of control measures. 
In addition, the final size method assumes that the proportion of susceptible persons is known at the start of an outbreak; however, the level of susceptibility to norovirus is not well known. Certain host genetic factors are associated with the ability of norovirus to establish an infection within a human host (38-41), leading to variable susceptibility to norovirus infection (42-44). Secretor-negative persons have nonfunctional fucosyltransferase-2 genes, causing infection failure for norovirus genogroups I and II type $4(38,40,41,45,46)$. Our estimates of $R_{0}$ and $\mathrm{R}_{\mathrm{e}}$ assume that $47 \%$ of the population in our dataset is susceptible at the start of all outbreaks. However, the proportion susceptible varies among outbreaks and potentially over time and age as the distribution of circulating norovirus genotypes change. Further, our regression model estimates were sensitive to our assumption of the percent susceptible at the start of an outbreak. When we assumed $47 \%$ and $80 \%$ of the population was susceptible, the estimated transmissibility of norovirus in private homes or residences and restaurants was higher than transmissibility in long-term care and assisted living facilities. However, when we assumed $27 \%$ of the population was susceptible at the start of an outbreak, the association between private homes or residences and restaurants reversed. These settings then had lower estimated transmission relative to outbreaks in long-term care and assisted living facilities because the population size that can be infected is much lower, thus reducing the estimates of $R_{0}$ and $R_{e}$. For example, if a household had 15 persons, the maximum possible $R_{0}$ assuming $27 \%$ susceptibility is 4 , which is lower than the average predicted $R_{0}$ for outbreaks in the reference group. Therefore, the results for private homes or residences and restaurants, where exposed population sizes are lower, should be interpreted with caution because transmission values in these settings might be underestimated. We also assumed that only symptomatic persons contribute to transmission in our calculation; persons with asymptomatic norovirus infections can contribute to transmission, but they likely are not as infectious as persons with symptomatic infections $(22,47)$.

Finally, our main analysis does not account for norovirus genotype. Because of the limited data available on outbreak genotype we were not able to fully assess whether certain genotypes were more transmissible. As more genotyping data become available, future studies should investigate transmissibility.

We estimated reproduction numbers by using the final size method for $>7,000$ outbreaks from a national outbreak reporting system, then used these estimates to examine factors associated with norovirus transmission. Our analyses suggest that norovirus transmission rates are modest. Such modest rates of $R_{e}$ suggest there are opportunities for effective control measures to curtail transmission of norovirus. However, challenges remain. Transmission by asymptomatic persons, which we did not account for in this analysis and generally goes undetected in surveillance, can limit the effectiveness of traditional control methods focused on ill persons, even for pathogens with modest transmission (48).

Overall, we found limited variation in $R_{0}$ and $R_{e}$ for reported norovirus outbreaks in the United States, particularly across different settings. Our findings highlight the need for better data on the total exposed population sizes in outbreaks, which heavily influence estimates of attack rates, $R_{0^{\prime}}$ and $R_{e^{\prime}}$ to further refine estimates of these outbreak factors.

This work was supported by funding from the Norovirus Vaccine Modeling (NoVaMod) project (grant no. R01 GM124280), the National Institutes of Health, Agency for Healthcare Research Quality (grant no. R01 HS025987), and the National Institutes of Health graduate and postdoctoral training grant in environmental health science and toxicology (grant no. T32 ES012870).

The findings and conclusions in this report are those of the authors and do not necessarily represent the official position of the Centers for Disease Control and Prevention or the US Department of Health and Human Services.

\section{About the Author}

Dr. Steele is an epidemiologist in the Influenza Division, National Center for Immunization and Respiratory Diseases, Centers for Disease Control and Prevention. Her research interests include infectious disease dynamics and predicting public health impacts of vaccination.

\section{References}

1. Hall AJ, Wikswo ME, Manikonda K, Roberts VA, Yoder JS, Gould LH. Acute gastroenteritis surveillance through the National Outbreak Reporting System, United States. Emerg Infect Dis. 2013;19:1305-9. https:/ / doi.org/10.3201/ eid1908.130482

2. Wikswo ME, Kambhampati A, Shioda K, Walsh KA, Bowen AB, Hall AJ. Outbreaks of acute gastroenteritis transmitted by person-to-person contact, environmental contamination, and unknown modes of transmission-United States, 2009-2013. MMWR Surveill Summ. 2015;64:1-16 https://doi.org/10.15585/mmwr.mm6412a1

3. U.S. Centers for Disease Control and Prevention. National Outbreak Reporting System (NORS) [cited 2018 Oct 22]. https://www.cdc.gov/nors/data.html

4. Burke RM, Shah MP, Wikswo ME, Barclay L, Kambhampati A, Marsh Z, et al. The norovirus epidemiologic 
triad: predictors of severe outcomes in US norovirus outbreaks, 2009-2016. J Infect Dis. 2019;219:1364-1372. https://doi.org/10.1093/infdis/jiy569

5. Rothman K, Greenland S, Lash T. Modern epidemiology. Philadelphia: Lippincott Williams \& Wilkins; 2008.

6. Keeling MJ, Rohani P. Modeling infectious diseases in humans and animals. Boston: Princeton University Press; 2007.

7. Diekmann O, Heesterbeeck JAP. Mathematical epidemiology of infectious diseases: model building, analysis and interpretation, 1st edition. West Essex, England: John Wiley \& Sons; 2000

8. Guerra FM, Bolotin S, Lim G, Heffernan J, Deeks SL, Li Y, et al. The basic reproduction number $\left(R_{0}\right)$ of measles: a systematic review. Lancet Infect Dis. 2017;17:e420-8. https:/ / doi.org/10.1016/S1473-3099(17)30307-9

9. Ridenhour B, Kowalik JM, Shay DK. Unraveling $\mathrm{R}_{0}$ : considerations for public health applications. Am J Public Health. 2014;104:e32-41. https:// doi.org/10.2105/ AJPH.2013.301704

10. Gaythorpe KAM, Trotter CL, Lopman B, Steele M, Conlan AJK. Norovirus transmission dynamics: a modelling review. Epidemiol Infect. 2018;146:147-58. https://doi.org/10.1017/S0950268817002692

11. U.S. Centers for Disease Control and Prevention. CaliciNet [cited 2020 Mar 18]. https:/ / www.cdc.gov/norovirus/ reporting/calicinet/data.html

12. Becker NG. Analysis of infectious disease data. London: Chapman and Hall/CRC; 1989.

13. Ajami NJ, Barry MA, Carrillo B, Muhaxhiri Z, Neill FH, Prasad BVV, et al. Antibody responses to norovirus genogroup GI.1 and GII.4 proteases in volunteers administered Norwalk virus. Clin Vaccine Immunol. 2012;19:1980-3. https://doi.org/10.1128/CVI.00411-12

14. Lindesmith L, Moe C, Lependu J, Frelinger JA, Treanor J, Baric RS. Cellular and humoral immunity following Snow Mountain virus challenge. J Virol. 2005;79:2900-9. https:/ / doi.org/10.1128/JVI.79.5.2900-2909.2005

15. Bernstein DI, Atmar RL, Lyon GM, Treanor JJ, Chen WH, Jiang $X$, et al. Norovirus vaccine against experimental human GII.4 virus illness: a challenge study in healthy adults. J Infect Dis. 2015;211:870-8. https:// doi.org/10.1093/infdis/ jiu497

16. Treanor JJ, Madore HP, Dolin R. Development of an enzyme immunoassay for the Hawaii agent of viral gastroenteritis. J Virol Methods. 1988;22:207-14. https:/ / doi.org/10.1016/0166-0934(88)90103-6

17. Frenck R, Bernstein DI, Xia M, Huang P, Zhong W, Parker S, et al. Predicting susceptibility to norovirus GII.4 by use of a challenge model involving humans. J Infect Dis. 2012;206:1386-93. https://doi.org/10.1093/infdis/jis514

18. Atmar RL, Opekun AR, Gilger MA, Estes MK, Crawford SE, Neill FH, et al. Norwalk virus shedding after experimental human infection. Emerg Infect Dis. 2008;14:1553-7. https://doi.org/0.3201/eid1410.080117

19. Leon JS, Kingsley DH, Montes JS, Richards GP, Lyon GM, Abdulhafid GM, et al. Randomized, double-blinded clinical trial for human norovirus inactivation in oysters by high hydrostatic pressure processing. Appl Environ Microbiol. 2011;77:5476-82. https:// doi.org/10.1128/AEM.02801-10

20. R Core Team. R: A language and environment for statistical computing. Vienna: R Foundation for Statistical Computing; 2018 [cited 2018 Oct 22]. http:/ / www.r-project.org

21. Blair G, Cooper J, Coppock A, Humphreys M, Sonnet L, Fultz N, et al. Package "estimatr". 2019 [cited 2019 Nov 12]. https://cran.r-project.org/web/packages/estimatr/ estimatr.pdf
22. Sukhrie FH, Teunis P, Vennema H, Copra C, Thijs Beersma MFC, Bogerman J, et al. Nosocomial transmission of norovirus is mainly caused by symptomatic cases. Clin Infect Dis. 2012;54:931-7. https://doi.org/10.1093/cid/cir971

23. Vanderpas J, Louis J, Reynders M, Mascart G, Vandenberg O. Mathematical model for the control of nosocomial norovirus. J Hosp Infect. 2009;71:214-22. https:/ / doi.org/ 10.1016/j.jhin.2008.11.024

24. Heijne JCM, Rondy M, Verhoef L, Wallinga J, Kretzschmar M, Low N, et al. Quantifying transmission of norovirus during an outbreak. Source Epidemiol. 2012;23:277-84. https:/ / doi.org/10.1097/EDE.0b013e3182456ee6

25. O'Dea EB, Pepin KM, Lopman BA, Wilke CO. Fitting outbreak models to data from many small norovirus outbreaks. Epidemics. 2014;6:18-29. https:/ / doi.org/ 10.1016/j.epidem.2013.12.002

26. Simmons K, Gambhir M, Leon J, Lopman B. Duration of immunity to norovirus gastroenteritis. Emerg Infect Dis. 2013;19:1260-7. https:/ / doi.org/10.3201/eid1908.130472

27. Steele MK, Remais JV, Gambhir M, Glasser JW, Handel A, Parashar UD, et al. Targeting pediatric versus elderly populations for norovirus vaccines: a model-based analysis of mass vaccination options. Epidemics. 2016;17:429. https:// doi.org/10.1016/j.epidem.2016.10.006

28. Milbrath MO, Spicknall IH, Zelner JL, Moe CL, Eisenberg JNS. Heterogeneity in norovirus shedding duration affects community risk. Epidemiol Infect. 2013;141:1572-84. https://doi.org/10.1017/S0950268813000496

29. Lawrence L, Kerrod E, Gani R, Leach S. Microbiological risk assessment for norovirus infection contribution to the overall burden afforded by foodborne infections. London: Food Standards Agency; 2004.

30. Ahmed SM, Lopman BA, Levy K. A systematic review and meta-analysis of the global seasonality of norovirus. PLoS One. 2013;8:e75922. https://doi.org/10.1371/journal.pone.0075922

31. Lopman B, Armstrong B, Atchison C, Gray JJ. Host, weather and virological factors drive norovirus epidemiology: time-series analysis of laboratory surveillance data in England and Wales. PLoS One. 2009;4:e6671.

https:/ / doi.org/10.1371/journal.pone.0006671

32. Duizer E, Bijkerk P, Rockx B, De Groot A, Twisk F, Koopmans M. Inactivation of caliciviruses. Appl Environ Microbiol. 2004;70:4538-43. https:/ / doi.org/10.1128/ AEM.70.8.4538-4543.2004

33 Doultree JC, Druce JD, Birch CJ, Bowden DS, Marshall JA. Inactivation of feline calicivirus, a Norwalk virus surrogate. J Hosp Infect. 1999;41:51-7. https:/ / doi.org/10.1016/ S0195-6701(99)90037-3

34. Hall AJ, Wikswo ME, Pringle K, Gould LH, Parashar UD; Division of Viral Diseases, National Center for Immunization and Respiratory Diseases, CDC. Vital signs: foodborne norovirus outbreaks - United States, 2009-2012. MMWR Morb Mortal Wkly Rep. 2014;63:491-5.

35. Shah MP, Wikswo ME, Barclay L, Kambhampati A, Shioda K, Parashar UD, et al. Near real-time surveillance of U.S. norovirus outbreaks by the norovirus sentinel testing and tracking network - United States, August 2009-July 2015. MMWR Morb Mortal Wkly Rep. 2017;66:185.

36. U.S. Centers for Disease Control and Prevention. Reporting and Surveillance for Norovirus. NoroSTAT [cited 2018 Oct 22]. https://www.cdc.gov/norovirus/reporting/ noroSTAT/index.html

37. Becker NG. Martingale methods for the analysis of epidemic data. Stat Methods Med Res. 1993;2:93-112. https://doi.org/10.1177/096228029300200106 
38. Hutson AM, Atmar RL, Marcus DM, Estes MK. Norwalk virus-like particle hemagglutination by binding to histo-blood group antigens. J Virol. 2003;77:405-15. https://doi.org/10.1128/jvi.77.1.405-415.2003

39. Hutson AM, Airaud F, Lependu J, Estes MK, Atmar RL. Norwalk virus infection associates with secretor status genotyped from sera. J Med Virol. 2005;77:116-20. https://doi.org/10.1128/jvi.77.1.405-415.2003

40. Hutson AM, Atmar RL, Graham DY, Estes MK. Norwalk virus infection and disease is associated with $\mathrm{ABO}$ histo-blood group type. J Infect Dis. 2002;185:1335-7. https://doi.org/10.1086/339883

41. Lindesmith L, Moe C, Marionneau S, Ruvoen N, Jiang X, Lindblad L, et al. Human susceptibility and resistance to Norwalk virus infection. Nat Med. 2003;9:548-53. https://doi.org/10.1038/nm860

42. Wyatt RG, Dolin R, Blacklow NR, DuPont HL, Buscho RF, Thornhill TS, et al. Comparison of three agents of acute infectious nonbacterial gastroenteritis by cross-challenge in volunteers. J Infect Dis. 1974;129:709-14. https://doi.org/10.1093/infdis/129.6.709

43. Parrino TA, Schreiber DS, Trier JS, Kapikian AZ, Blacklow NR. Clinical immunity in acute gastroenteritis caused by Norwalk agent. N Engl J Med. 1977;297:86-9. https:/ / doi.org/10.1056/NEJM197707142970204

44. Graham DY, Jiang X, Tanaka T, Opekun AR, Madore HP, Estes MK. Norwalk virus infection of volunteers: new insights based on improved assays. J Infect Dis. 1994;170:3443. https://doi.org/10.1093/infdis/170.1.34

45. Marionneau S, Airaud F, Bovin NV, Pendu JL, Ruvoën-Clouet N. Influence of the combined ABO, FUT2, and FUT3 polymorphism on susceptibility to Norwalk virus attachment. J Infect Dis. 2005;192:1071-7. https://doi.org/10.1086/432546

46. Marionneau S, Ruvoën N. BeatriceLe Moullac-Vaidye, Monique Clement, Anne Cailleau-Thomas,

Guillermo Ruiz-Palacois, et al. Norwalk virus binds to histo-blood group antigens present on gastroduodenal epithelial cells of secretor individuals. Gastroenterology. 2002;122:1967-77. https://doi.org/10.1053/ gast. 2002.33661

47. Sukhrie FH, Siebenga JJ, Beersma MFC, Koopmans M. Chronic shedders as reservoir for nosocomial transmission of norovirus. J Clin Microbiol. 2010;48:4303-5. https:/ / doi.org/10.1128/JCM.01308-10

48. Ferguson NM, Cummings DAT, Fraser C, Cajka JC, Cooley PC, Burke DS. Strategies for mitigating an influenza pandemic. Nature. 2016;442:448-52. https://doi.org/10.1038/nature04795

Address for correspondence: Molly Steele, Centers for Disease Control and Prevention, 1600 Clifton Rd NE, Mailstop H24-7; Atlanta, GA 30329-4027, USA; email: MSteele@cdc.gov

\section{Tickborne Ehrlichia in North Carolina}

While caring for patients in North Carolina, Dr. Ross Boyce began to suspect that tickborne Ehrlichia was being underdiagnosed. His study showed that Ehrlichia, despite being relatively common, was only tested for in about a third of patients thought to have a tickborne illness.

In this EID podcast, Dr. Ross Boyce, an infectious disease physician at the University of North Carolina at Chapel Hill, examines the prevalence and diagnosis of Ehrlichia in North Carolina.

Visit our website to listen: https: / / go.usa.gov/ xy6UH
EMERENG INFECTIOUS DISEASES 